\title{
Application of percutaneous vertebroplasty in the treatment of multiple thoracic metastases
}

\author{
WENHUA LIU, SHIJUN ZHOU and SHAOFENG WANG
}

Department of Spinal Surgery, The Affiliated Hospital of Weifang Medical College, Weifang, Shandong 261031, P.R. China

Received April 29, 2014; Accepted January 29, 2015

DOI: $10.3892 / \mathrm{ol} .2015 .3145$

\begin{abstract}
The present study aimed to explore the clinical implications of percutaneous vertebroplasty (PVP) in the treatment of multiple thoracic metastases. A total of 104 involved vertebrae of 28 patients with multiple thoracic metastases underwent PVP and the injection of bone cement. The pain relief rate and activity of daily life were assessed, and the morphological changes of the involved vertebral bodies and the invasiveness of the intraspinal tumor were also observed at 1 week, and 3, 6 and 12 months post-surgery, respectively. The pain relief rate increased at each time-point following PVP. The quality of life scores were also significantly higher than the pre-operative scores. After 12 months of follow-up, the post-operative heights of the anterior border, center and posterior border of the vertebral bodies were not noted to be statistically different from those prior to PVP ( $>0.05)$. The present study concluded that PVP has a good analgesic effect on multiple thoracic metastases. PVP not only improves the quality of life of patients significantly, but also prevents further vertebral collapse and the invasiveness of intraspinal tumors, avoiding the nerve dysfunction caused by spinal cord compression.
\end{abstract}

\section{Introduction}

Bone is one of the most common sites of metastasis and the spine is the most frequently affected painful skeletal site (1). Spinal metastases are observed in more than two-thirds of patients who succumb to cancer. Pathological fractures therefore affect vertebrae in 10-20\% of cases that involve the posterior wall of the vertebral body, causing them to protrude posteriorly and compromise the spinal canal, resulting in neurological injury (2). The most common sites of disease are the thoracic vertebrae (60-80\%), followed by the lumbar $(20 \%)$ and cervical (10\%) spine (3).

Correspondence to: Dr Shaofeng Wang, Department of Spinal Surgery, The Affiliated Hospital of Weifang Medical College, 2,428 Yuhe Road, Kuiwei, Weifang, Shandong 261031, P.R. China E-mail: shaofengwangcn@126.com

Key words: percutaneous vertebroplasty, thoracic vertebra, multiple metastases, bone cement
The invasiveness of metastatic lesions in the vertebral body and its attachments may cause severe thoracic lumbar and back pain, and even neurological dysfunction, reducing the patient's quality of life and hastening mortality. Radiotherapy weakens the bone remodeling ability and increases the risk of vertebral collapse and nerve compression (4-6). By contrast, surgery is more suitable for patients with an oppressed spinal cord, but this causes more trauma and has a high incidence of complications; in particular, corpectomy is not suitable for patients with multiple vertebral metastases. Percutaneous vertebroplasty (PVP) has been widely used in the treatment of vertebral osteolytic tumors, and has become the main treatment of these diseases $(1,7)$; however, its application in the treatment of multiple thoracic metastases is rarely reported.

In the present study, the clinical data of 28 patients with multiple thoracic metastases who received PVP at the Department of Spinal Surgery at the Affiliated Hospital of Weifang Medical College (Weifang, Shangdong, China), between March 2006 and March 2008, were therefore retrospectively analyzed in order to explore the treatment effectiveness of the injection of bone cement on multiple thoracic metastases.

\section{Materials and methods}

General data. A total of 28 patients with multiple thoracic metastases, consisting of 9 males and 19 females, aged 36-76 years (mean, 60.5 years), were retrospectively analyzed. The study was conducted in accordance with the Declaration of Helsinki and with the approval of the Ethics Committee of Weifang Medical College. Written informed consent was obtained from all participants. All patients presented with thoracolumbar and back pain, percussion pain and movement disorders of the thoracolumbar vertebrae, among which, one patient presented with decreased superficial sensation of the skin of one lower limb. X-ray revealed various degrees of damage and/or collapse in the involved vertebral bodies. Computed tomography (CT) and magnetic resonance imaging (MRI) scans revealed osteolytic bone destruction, but the posterior wall of the involved vertebral body was intact (Fig. 1). Emission CT (ECT) revealed significant radioactivity accumulation in the thoracic lesions. No cases were complicated with severe bleeding, coagulation disorders or severe lung diseases. Patients with advanced cachexia and extreme physical weakness, as well as patients with spinal cord involvement and paraplegia, were excluded. In total, the 


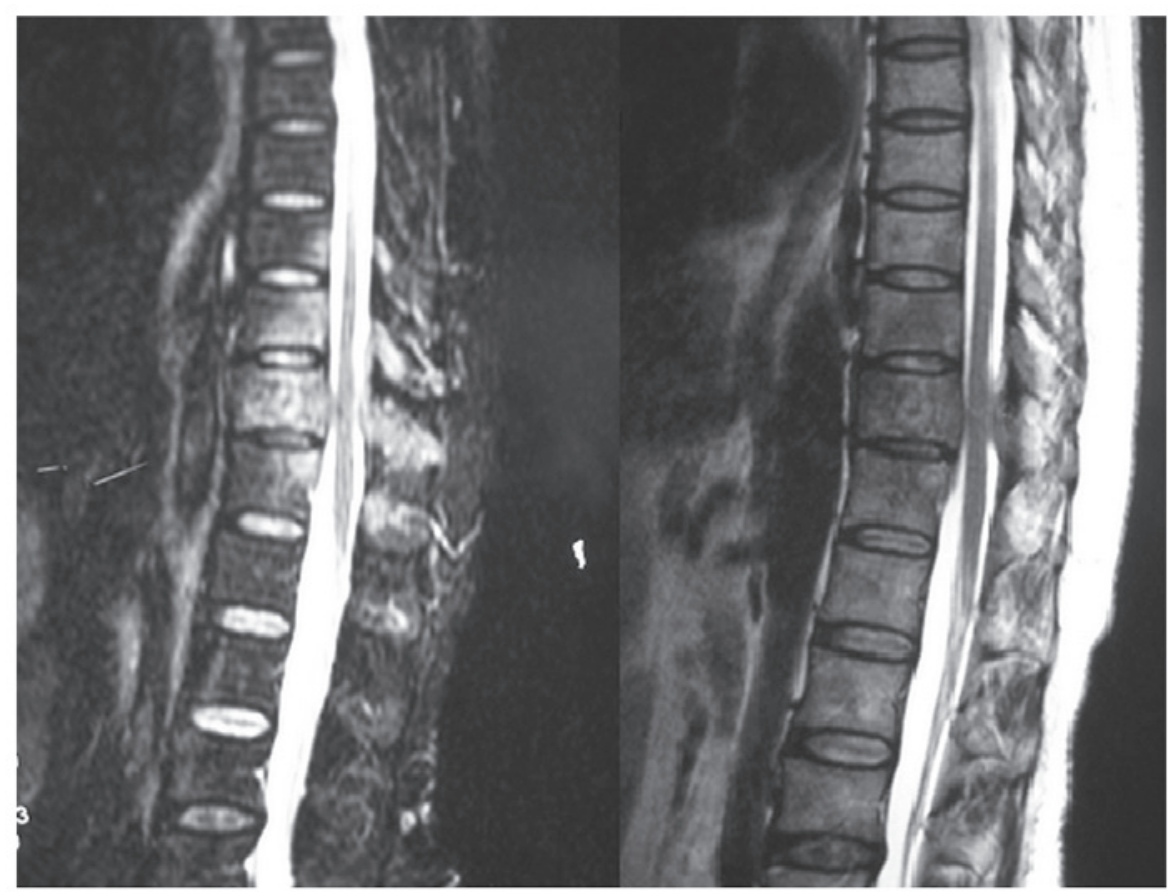

Figure 1. Pre-operative magnetic resonance imaging scan revealing vertebral metastases at the T9, T10, T11 and T12 vertebrae. The T11 vertebral body was osteolytically destroyed, but its posterior wall was intact. Left image, T2-weighted fat-saturated imaging. Right image, pure T2-weighted imaging.

28 patients exhibited 104 involved vertebral bodies, spread amongst the T12 (n=16), T11 (n=21), T10 (n=23), T9 (n=19), T8 $(n=11)$, T7 $(n=6)$, T6 $(n=3)$, T5 $(n=1)$, T4 $(n=3)$ and T2 $(n=1)$ vertebrae. Among the 28 patients, there were 11 patients with continuous thoracic metastases and 17 patients with interval metastases. The number of involved thoracic vertebrae in each patient was between two and six. There were six thoracic vertebrae involved in three cases, five thoracic vertebrae involved in four cases, four thoracic vertebrae involved in 11 cases, three thoracic vertebrae involved in six cases and two thoracic vertebrae involved in four cases. The sources of metastases included breast cancer $(n=12)$, prostate cancer $(n=3)$, gastric cancer $(n=3)$, lung cancer $(n=3)$, kidney cancer $(n=1)$, liver cancer $(n=2)$ and thyroid carcinoma $(n=1)$. There were two cases with unknown origin. A total of 16 cases of primary lesions had been surgically removed and determined histologically, 10 cases were confirmed using needle biopsy and endoscopy, and the other two had unknown origin.

Methods. Patients were asked to maintain a prone position. The lower thoracic spine was punctured through the pedicle of the vertebral arch, and the upper thoracic spine was also punctured through the pedicle of the vertebral arch or through the gap between the costal head and the vertebral body. Guided by the G-arm X-ray machine, the needle (Guanlong Medical Supplies Co., Jinan, China) was percutaneously inserted into the pedicle of the vertebral arch at a $10-15^{\circ}$ angle, and further passed into the anterior third of the vertebral body. The point of the needle was located in the upper or lower half of the vertebral body according to the location of the tumor determined by MRI. For the two patients with severe osteoporosis, in order to avoid the difficulties of the G-arm X-ray machine in positioning the involved vertebra and the needle insertion point, the puncture was performed with the guidance of a large digital subtraction angiography machine (Angiostar, Siemens, Munich, Germany). Bone cement (Heraeus Medical GmbH, Wehrheim, Germany), which can be developed under X-ray, was prepared with a ratio of powder $(\mathrm{g})$ to liquid $(\mathrm{m} 1)$ of $2: 1$. With the guidance of the X-ray, the bone cement in starch paste state was fractionatedly and slowly injected into the involved vertebral bodies at a volume of 0.5 to $1.0 \mathrm{ml}$ per time until the distribution of bone cement reached the edge of the vertebral body. If any apparent bone cement leakage was observed at the side or rear of the vertebral body, the injection was immediately stopped (Fig. 2). The total injection volume was 2.0 to $2.5 \mathrm{ml}$ for the upper thoracic spine and 2.5 to $3.5 \mathrm{ml}$ for the lower thoracic spine. During the injection of bone cement, patients were monitored by electrocardiogram and the sensory and movement changes of their lower extremity was observed at all time. Post-operative application of antibiotics was administered for three days. Patients were allowed to ambulate after $48 \mathrm{~h}$ of bed rest. Periodic post-operative reviews of the distribution of bone cement in the vertebral bodies were performed using X-ray or CT scans (Fig. 3). Following PVP, all patients received appropriate chemotherapy and radiotherapy, with schemes specific for each patient (Table I).

Evaluation of therapeutic effect. To assess pain relief, the pain status of the patients prior to and following treatment was measured according to visual analog scales (8). Post-operative pain relief was graded into six levels: Grade 0, no relief; grade I, pain reduced by $<25 \%$; grade II, pain reduced by $25-50 \%$ (the dosage of analgesics should be reduced); grade III, pain reduced by $51-75 \%$ (the dosage of analgesics should be reduced by one step); grade IV, pain reduced by $76-90 \%$ (the dosage of analgesics should be reduced by two steps or the administration should be stopped); and grade $\mathrm{V}$, complete pain relief (the analgesics 


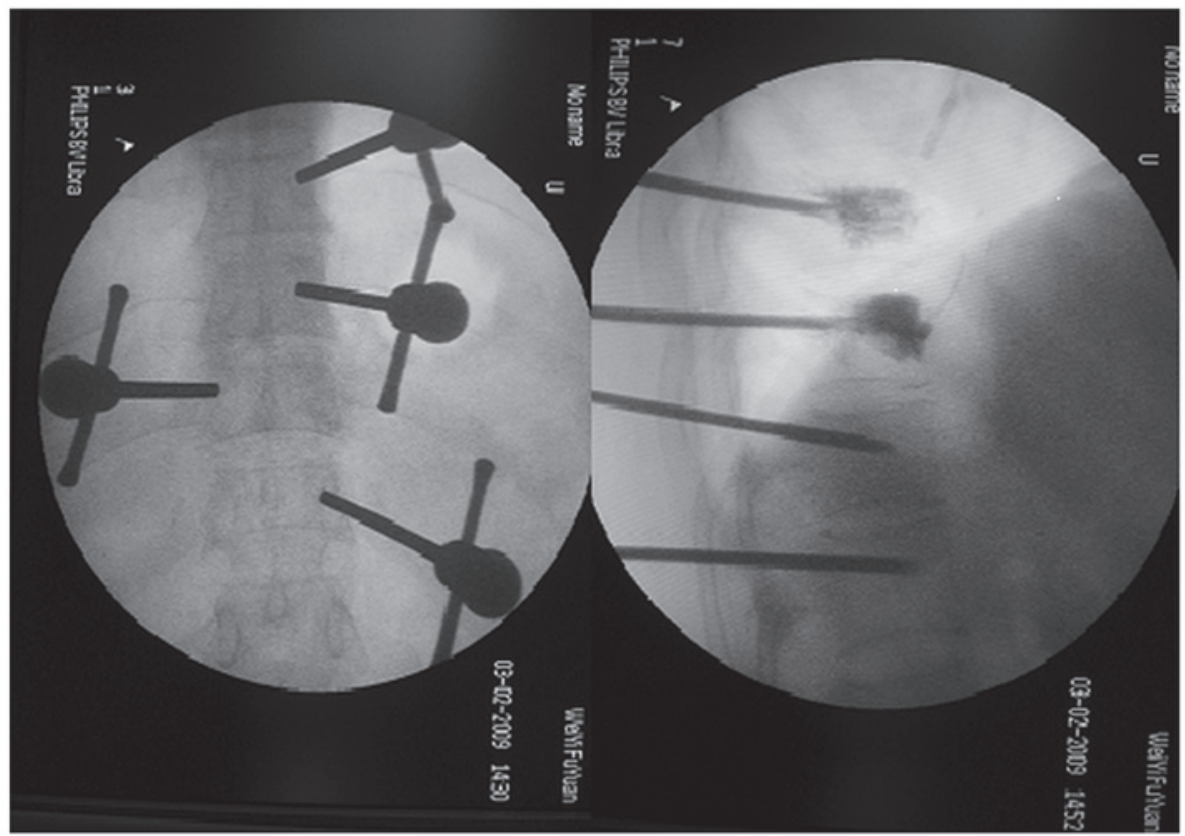

Figure 2. Bone cement is injected into the vertebral body through the puncture needle inserted through the pedicle of the vertebral arch with the guidance of the G-arm X-ray.

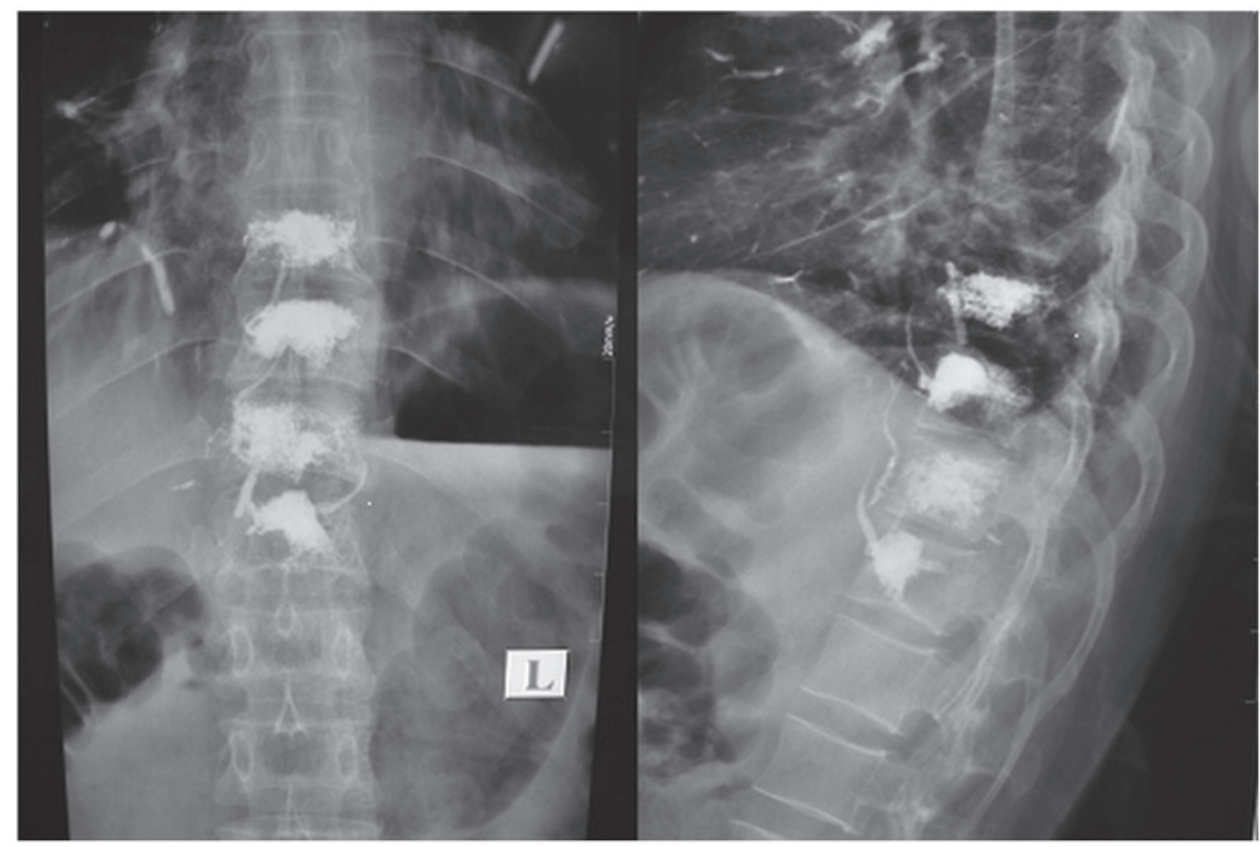

Figure 3. X-ray revealing the good distribution and full filling of bone cement in the vertebral body following surgery.

should be stopped). Pain relief assessment was conducted at 1 week, and 3,6 and 12 months post-PVP. Grades IV and V were considered as excellent, grade III as good, grade II as a response and grades I and 0 as no response.

To assess quality of life, ratings of the activity of daily life (ADL) (9) were used.

To assess the morphological changes of the involved vertebrae and the invasiveness of the intraspinal tumors, the heights of the anterior border, center and posterior border of the involved vertebrae were measured using X-ray examination one year after PVP surgery to determine whether vertebral body collapse had occurred. The attachments of the involved vertebrae, the tumor invasion in the vertebral canal and the oppression of the spinal cord were also observed using a CT or MRI scan.

Statistical analysis. Statistical analyses were performed independently by a non-clinical research assistant and an outside party to ensure objectivity using SPSS version 16.0 software (SPSS, Inc., Chicago, IL, USA). Student's t-test was used and $\mathrm{P} \leq 0.05$ was considered to indicate a statistically significant result for continuous variables. 
Table I. Radiotherapy and chemotherapy treatments for patients with percutaneous vertebroplasty.

\begin{tabular}{llrl}
\hline Patient tumor type & \multicolumn{1}{c}{ Chemotherapy } & Radiotherapy & Other treatments \\
\hline Breast cancer & TXT+EPI & Local radiotherapy & Endocrine therapy \\
Prostate cancer & DDP+VP-16 & \\
Gastric cancer & L-OHP+5-FU+CF & \\
Small cell lung cancer & CBP+VP-16 & \\
Non-small cell lung cancer & GEMZ+DDP & \\
Kidney cancer & GEMZ & Local radiotherapy \\
Liver cancer & Regional 5-FU perfusion & \\
Thyroid carcinoma & & Symptomatic therapy \\
Unknown origin & & \\
\hline TXT, taxotere; EPI, epirubicin; DDP, cis-diamminedichloroplatinum; VP-16, vepesid; L-OHP, L-oxaliplatin; 5-FU, 5-fluoro- \\
uracil; CF, calcium folinate; CBP, carboplatin; GEMZ, gemcitabine. & \\
\hline
\end{tabular}

Table II. Post-operative pain relief and its response rate in patients treated for multiple thoracic metastases.

\begin{tabular}{lcccc}
\hline Pain relief grades & 1 week & 3 months & 6 months & 12 months \\
\hline Grade V, n & 8 & 11 & 9 & 7 \\
Grade IV, n & 8 & 9 & 5 & 6 \\
Grade III, n & 7 & 2 & 5 & 10 \\
Grade II, n & 3 & 1 & 2 & 1 \\
Grade I, n & 2 & 0 & 1 & 1 \\
Grade 0, n & 0 & 0 & 1 & 1 \\
Mortalities, n & 0 & $89.3(25 / 28)$ & $81.5(22 / 27)$ & $88.5(23 / 26)$ \\
Response rate, $\mathrm{n}(\%)$ & $82.1(23 / 28)$ & & & 1 \\
\hline
\end{tabular}

\section{Results}

The 28 patients all received successful punctures, including 4 bilateral thoracic pedicle punctures and 100 unilateral thoracic vertebral punctures. Bone cement leakage occurred in one thoracic vertebral body in the intervertebral space. The average surgical time for each individual vertebra was $0.5 \mathrm{~h}$ (range, 0.3-0.9 h). The total injection volume of bone cement was 2.2 to $3.7 \mathrm{ml}$ (mean, $3.1 \pm 0.4 \mathrm{ml}$ ). No spinal cord compression was observed, nor were other complications, such as pulmonary embolism and infection. Patients were followed up for 6 to 24 months (mean, 14 months). One patient succumbed at 6 months post-surgery and another succumbed at 12 months post-surgery. All mortalities were due to systemic metastases of malignant tumors.

Pain relief. Post-operative pain was graded according to the degree of pain relief. The pain relief rates of the patients at 1 week, and 3, 6 and 12 months post-PVP were 82.1, 89.3, 81.5 and $88.5 \%$, respectively (Table II).

$A D L$. At the end of follow-up, the ADL scores of the remaining 26 patients were significantly different from those prior to surgery $(\mathrm{P}<0.01)$. The ability to use the toilet, move, walk horizontally for $50 \mathrm{~m}$, and go up and down the stairs was significantly improved compared with prior to surgery. In addition, the ability to eat and dress were also statistically different prior to and following surgery (Table III).

Vertebral height. One year after PVP, the heights of the anterior border, center and posterior border of the 98 involved vertebrae of the remaining 26 patients were not significantly different from those prior to PVP (P>0.05; Table IV). As demonstrated by the pre-operative CT and MRI scan one year after PVP, there were five vertebrae in four patients that presented with pedicle invasiveness and extending into the spinal canal, but no obvious neurological symptoms were observed.

\section{Discussion}

Spinal malignant tumors are mainly metastases. It has been reported that 60 to $80 \%$ of malignant tumors metastasize to the spine, mainly involving the thoracic vertebrae, followed by the lumbar and then the cervical vertebrae (1-3). The invasiveness of the metastasis to the vertebral body and its accessories leads to severe thoracolumbar and back pain, and even neurological dysfunction, seriously affecting a patient's daily life. However, conventional radiotherapy and chemotherapy achieve a less than ideal therapeutic effect. Although the effect of active 
Table IV. Comparison of vertebral heights of the 98 involved vertebrae of the 26 patients prior to $(n=26)$ and 1 year after $(n=26)$ surgery.

\begin{tabular}{lccc}
\hline Time-points & Anterior border & Center & Posterior border \\
\hline Prior to PVP & $18.7 \pm 1.6$ & $17.9 \pm 1.9$ & $19.9 \pm 2.0$ \\
1 year after PVP & $19.1 \pm 1.7$ & $17.8 \pm 1.5$ & $19.8 \pm 1.8$ \\
t-value & 1.452 & 1.637 & 1.836 \\
P-value & 0.095 & 0.121 & 0.134 \\
\hline
\end{tabular}

Data are presented as the mean \pm standard deviation.

surgical treatment in recent years has been improved greatly and the surgical indications have also been expanded along with the improvement of spinal surgery techniques, treating multiple thoracic metastases remains a challenge, particularly for interval metastases $(10,11)$. Since PVP was applied in the treatment of spinal metastases by Kaemmerlen et al (12) in 1989, good results have been achieved in China and abroad. However, its application in the treatment of multiple thoracic metastases has never been reported.

Metastasis destroys the vertebral body by causing micro-fractures and compression fractures, resulting in spinal instability (13). The nerve endings inside and outside the vertebral body are stimulated and damaged, which is the most common reason for thoracolumbar and back pain $(14,15)$. The direct invasiveness of the tumor tissues to the nerve endings also causes pain (16). The present study showed that PVP quickly relieved thoracolumbar and back pain in patients with multiple thoracic metastases. The mechanisms for this may be as follows: i) The stable and supportive roles of polymethylmethacrylate (PMMA) on the vertebral body. The PMMA injected into the involved vertebrae solidifies into clumps in a short time, inhibiting the reduction of the support force caused by vertebral destruction and fixing the micro-fractures in the involved vertebral bodies, thereby reducing the stimulation on the nerve roots and sinus vertebral nerve due to the loss of stability of the spine $(17,18)$. ii) The thermogenic effect during the polymerization of PMMA. The polymerization of PMMA increases the local temperature of the vertebral body, reaching 52 to $93^{\circ} \mathrm{C}$, which leads to degeneration, necrosis and a loss of sensation in the pain nerve endings within the vertebral body. Furthermore, the heat effectively inactivates the tumor cells and reduces the production of the mediators of inflammation and pain. The heat also prevents the growth of cancer cells and reduces its compression on the nerve endings (19). iii) The monomer toxicity of PMMA. PMMA produces monomers with toxic side-effects in the body, causing peripheral nerve endings and tumor cell necrosis (20).

Simple chemotherapy or radiotherapy achieves only limited results on spinal metastases. Moreover, it cannot relieve the unstable spine and spinal cord compression caused by the invasiveness of tumors $(15,21,22)$. In the present study, PVP significantly prevented the involved vertebral bodies from further collapse or invasion into the spinal canal, and then prevented it from nerve dysfunction induced by spinal 
cord compression. Since the destruction of metastatic tumors is mostly osteolytic, it often results in bone defects. The vertebral compression fracture occurs when the spine bears the body weight and consequently increases pain and even causes neurological symptoms $(5,6,19)$. The injection of bone cement can strengthen the vertebral structure and restore the height, proof pressure and intensity of vertebral bodies. In addition, the heat and monomers produced locally have antitumor effects, which reduce the local tumor burden and consequently reduce the destruction of local tumors on bones (18). All these effects significantly prevent the vertebral bodies from further collapse and from invasion of the spinal tumors.

Currently, the evaluation of the treatment efficacy of PVP on vertebral metastases is mainly based on pain relief. However, the simple application of pain relief assessment cannot fully reflect the patient's quality of life. In the present study, ADL scores were used to comprehensively assess quality of life following PVP. The results indicated that the application of PVP in the treatment of multiple thoracic metastases comprehensively improves a patient's quality of life.

The major complication of PVP is the leakage of bone cement to the surrounding area, causing mechanical compression on nerve roots and the spinal cord or simultaneously causing thermal damage. The leakage of bone cement into the peripheral veins, particularly into the basivertebral venous plexus, can cause a pulmonary embolism $(17,20)$. The occurrence of complications is associated with the injection rate of bone cement. Thus, the injection of bone cement should be performed slowly with the guidance of X-ray. Side monitoring can prevent bone cement permeating into the spinal canal, and anteroposterior monitoring can prevent bone cement leaking bilaterally into the intervertebral foramen. In order to avoid leakage, bone cement should be injected fractionatedly at a volume of 0.5 to $1.0 \mathrm{ml}$ each time. If there are any signs of leakage, the injection should be immediately stopped. The puncture needle should be inserted into the anterior third of the vertebral body, but not wear through the inner wall of the pedicle. The consistency of bone cement for infusion is also a significant factor. Its flow is difficult to control if it is too thin, as it leaks easily and also refluxes with venous flow. Moreover, a careful study of the preoperative imaging data is also critical to prevent leakage.

Compared with surgical ablation of thoracic metastases, PVP is less harmful to patients and is relatively easy to administer; it effectively relieves the pain caused by osteolytic vertebral metastases, increases vertebral strength and improves spinal stability. Moreover, PVP can significantly prevent the invasion of spinal tumors and improve the patient's quality of life. This innovative technology for micro-spinal surgery is likely to become one of the primary means of palliative treatment for multiple thoracic metastases.

\section{Acknowledgements}

This study was supported by a grant from the Special Training Fund for Domestic and Abroad High-level Health Technological Talents in Shandong Province (grant no. 2013WS0277).

\section{References}

1. Barragán-Campos HM, Vallée JN, Lo D, et al: Percutaneous vertebroplasty for spinal metastases: complications. Radiology 238: 354-362, 2006.

2. Masala S, Anselmetti GC, Muto M, Mammucari M, Volpi T and Simonetti G: Percutaneous vertebroplasty relieves pain in metastatic cervical fractures. Clin Orthop Relat Res 469: 715-722, 2011.

3. Thanos L, Mylona S, Galani P, et al: Radiofrequency ablation of osseous metastases for the palliation of pain. Skeletal Radiol 37: 189-194, 2008.

4. Clarençon F, Jean B, Pham HP, et al: Value of percutaneous radiofrequency ablation with or without percutaneous vertebroplasty for pain relief and functional recovery in painful bone metastases. Skeletal Radiol 42: 25-36, 2013.

5. Anselmetti GC, Marcia S, Saba L, et al: Percutaneous vertebroplasty: multi-centric results from EVEREST experience in large cohort of patients. Eur J Radiol 81: 4083-4086, 2012.

6. Masala S, Massari F, Fiori R, Mammucari M, Bartolucci DA and Simonetti G: Future directions in percutaneous vertebroplasty. Radiol Med 114: 976-983, 2009.

7. Anselmetti GC, Corgnier A, Debemardi F and Regge D: Treatment of painful compression vertebral fractures with vertebroplasty: results and complications. Radiol Med 110: 262-272, 2005.

8. Burton AW, Reddy SK, Shah HN, Tremont-Lukats I and Mendel E: Percutaneous vertebroplasty - a technique to treat refractory spinal pain in the setting of advanced metastatic cancer: a case series. J Pain Symptom Manage 30: 87-95, 2005.

9. Lips P and van Schoor NM: Quality of life in patients with osteoporosis. Osteoporos Int 16: 447-455, 2005.

10. Simmons ED and Zheng Y: Vertebral tumors: surgical versus nonsurgical treatment. Clin Orthop Relat Res 443: 233-247, 2006.

11. Lee B, Franklin I, Lewis JS, Coombes RC, Leonard R, Gishen P and Stebbing J: The efficacy of percutaneous vertebroplasty for vertebral metastases associated with solid malignancies. Eur J Cancer 45: 1597-1602, 2009.

12. Kaemmerlen P, Thiesse P, Bouvard H, Biron P, Mornex F and Jonas P: Percutaneous vertebroplasty in the treatment of metastases. Technic and results. J Radiol 70: 557-562, 1989 (In French).

13. Oakland RJ, Furtado NR, Timothy J and Hall RM: The biomechanics of vertebroplasty in multiple myeloma and metastatic bladder cancer: a preliminary cadaveric investigation. J Neurosurg Spine 9: 439-501, 2008.

14. Do HM, Kim BS, Marcellus ML, Curtis L and Marks MP: Prospective analysis of clinical outcomes after pereutaneous vertebroplasty for painful osteoporotic vertebral body fractures. AJNR Am J Neuroradiol 26: 1623-1628, 2005.

15. Chen LH, Hsieh MK, Niu CC, Fu TS, Lai PL and Chen WJ: Percutaneous vertebroplasty for pathological vertebral compression fractures secondary to multiple myeloma. Arch Orthop Trauma Surg 132: 759-764, 2012.

16. Mao HQ, Yang HL, Geng DC, Bao ZH and Tang TS: Spinal extradural arachnoid cyst following percutaneous vertebroplasty. Eur Spine J 20 (Suppl 2): S206-S210, 2011.

17. Trumm C, Jakobs T, Pahl A, et al: CT fluoroscopy-guided percutaneous vertebroplasty in patients with multiple myeloma: analysis of technical results from 44 sessions with 67 vertebrae treated. Diagn Interv Radiol 18: 111-120, 2012.

18. Kallmes DF, Comstock BA, Heagerty PJ, et al: A randomized trial of vertebroplasty for osteoporotic spinal fractures. New Engl J Med 361: 569-579, 2009.

19. Garland P, Gishen P and Rahemtulla A: Percutaneous vertebroplasty to treat painful myelomatous vertebral deposits-long-term efficacy outcomes. Ann Hematol 90: 95-100, 2011.

20. Yan D, Duan L, Li J, Soo C, Zhu H and Zhang Z: Comparative study of percutaneous vertebroplasty and kyphoplasty in the treatment of osteoporotic vertebral compression fractures. Arch Orthop Trauma Surg 131: 645-650, 2011.

21. Brown DB, Gilula LA, Sehgal M and Shimony JS: Treatment of chronic symptomatic vertebral compression fracture with percutaneous vertebroplasty. AJR Am J Roentgenol 182: 319-322, 2004

22. Jacofsky DJ, Papagelopoulos PJ and Sim FH: Advances and challenges in the surgical treatment of metastatic bone disease. Clin Orthop Relat Res 415 (Suppl): S14-S18, 2003. 